\title{
Electron and Nucleon Localization Functions of Oganesson: Approaching the Thomas-Fermi Limit
}

\author{
Paul Jerabek, ${ }^{1}$ Bastian Schuetrumpf, ${ }^{2}$ Peter Schwerdtfeger, ${ }^{1,3}$ and Witold Nazarewicz ${ }^{4}$ \\ ${ }^{1}$ Centre for Theoretical Chemistry and Physics, The New Zealand Institute for Advanced Study, \\ Massey University Auckland, 0632 Auckland, New Zealand \\ ${ }^{2}$ NSCL/FRIB Laboratory, Michigan State University, East Lansing, Michigan 48824, USA \\ ${ }^{3}$ Centre for Advanced Study (CAS) at the Norwegian Academy of Science and Letters, Drammensveien 78, NO-0271 Oslo, Norway \\ ${ }^{4}$ Department of Physics and Astronomy and FRIB Laboratory, Michigan State University, East Lansing, Michigan 48824, USA
}

(Received 27 July 2017; revised manuscript received 2 December 2017; published 31 January 2018; corrected 24 January 2020)

\begin{abstract}
Fermion localization functions are used to discuss electronic and nucleonic shell structure effects in the superheavy element oganesson, the heaviest element discovered to date. Spin-orbit splitting in the $7 p$ electronic shell becomes so large $(\sim 10 \mathrm{eV})$ that $\mathrm{Og}$ is expected to show uniform-gas-like behavior in the valence region with a rather large dipole polarizability compared to the lighter rare gas elements. The nucleon localization in $\mathrm{Og}$ is also predicted to undergo a transition to the Thomas-Fermi gas behavior in the valence region. This effect, particularly strong for neutrons, is due to the high density of single-particle orbitals.
\end{abstract}

DOI: 10.1103/PhysRevLett.120.053001

Introduction.-Oganesson $(Z=118)$ is the recent addition to the periodic table of the elements and the chart of nuclides [1]. The isotope ${ }_{118}^{294} \mathrm{Og}$ was produced in a heavy ion fusion reaction with a ${ }_{20}^{48} \mathrm{Ca}$ beam and a ${ }_{98}^{249} \mathrm{Cf}$ target $[2,3]$. The heaviest element studied chemically to date is Fl $(Z=114)$. Its relatively long half-life, $1-2 \mathrm{~s}$, enables chemical studies with $\sim 5$ atoms/day, which marks the limit of chemistry today $[4,5]$. The estimated $\alpha$-decay halflife of ${ }_{118}^{294} \mathrm{Og}, 0.89_{-0.31}^{+1.07} \mathrm{~ms}$, is too short for chemical "oneatom-at-a-time" studies; hence, its chemical properties must be inferred from advanced atomic calculations based on relativistic quantum theory [6-19]. According to these, Og has a closed-shell $[\mathrm{Rn}] 5 f^{14} 6 d^{10} 7 s^{2} 7 p^{6}$ configuration $[13,20,21]$, with a very large spin-orbit splitting of the $7 p$ shell $(9.920 \mathrm{eV}$ at the Dirac-Breit-Hartree-Fock and $10.125 \mathrm{eV}$ at the Fock-space coupled-cluster level; see below). In contrast to its electronic configuration ( $\mathrm{Og}$ completes the seventh period of the periodic table), it is not expected to behave like a typical rare gas of group 18 . For example, the relativistic $7 p_{3 / 2}$ expansion and the relativistic $8 s$ contraction make $\mathrm{Og}$ the first rare gas element with a positive electron affinity of $0.064 \mathrm{eV}$ $[10,16,22]$. This result includes a substantial quantum electrodynamic correction of $0.006 \mathrm{eV}$ [16].

Nuclear structure calculations predict ${ }^{294} \mathrm{Og}$ to be a deformed nucleus [23-26], eight neutrons away from the next neutron shell closure at ${ }^{302} \mathrm{Og}(N=184)$ [27-32]. A new factor impacting properties of superheavy nuclei is the strong electrostatic repulsion: The Coulomb force in superheavy nuclei cannot be treated as a small perturbation atop the dominating nuclear interaction; the resulting polarization effects due to Coulomb frustration are expected to influence significantly the proton and neutron distributions and shell structure [26,28,31,33-37]. In particular, the isotope ${ }_{118}^{294} \mathrm{Og}$ is believed to be a semibubble system with a sizable central depression of the proton density [26].

The objective of this Letter is to study the electronic and nucleonic shell structure of superheavy elements. The electronic shell structure is expected to be impacted by the transition from the $L S$ coupling of the Schrödinger equation at lower atomic numbers to the $j j$ coupling of the Dirac equation at large $Z$ values. In the nuclear case, the shell structure is expected to be washed out due to the large density of single-nucleonic states. While the kinematics of protons and neutrons in a nucleus is nonrelativistic, the large spin-orbit coupling (that is about an order of magnitude greater than in the atomic case due to large spindependent components of the nucleon-nucleon interaction [38]) results in a $j j$ coupling. Therefore, for both electronic and nucleonic systems, the pattern of single-particle levels of superheavy species is expected to be strongly impacted by both radial and total angular momentum characteristics $[39,40]$. To describe these changes quantitatively, we utilize the fermion localization measure [41], which is an excellent indicator of shell structure. In particular, we investigate the transition from the regime of strong localization, governed by shell effects, to a more delocalized regime typical of a uniform-density Thomas-Fermi gas. As we shall demonstrate, superheavy species constitute an excellent territory to look for such a transition.

Fermion localization function.-The spatial localization measure was originally proposed in atomic and molecular physics to characterize shell structure and chemical bonding in electronic systems [41-47]. It has been subsequently introduced to nuclear physics to visualize cluster structures 
in light nuclei [48]. The novel nuclear applications include a description of nuclear fission [49] and heavy-ion fusion [50] and nucleonic matter in the inner crust of neutron stars [51]. In electronic systems, the spatial localization function is referred to as the electron localization function (ELF), and in nuclear systems as the nucleon localization function (NLF). It is based on the inverse of the conditional probability of finding a fermion of type $q(=e, n$, or $p)$ in the vicinity of another fermion of the same type and same spin or signature quantum number $\sigma(=\uparrow$ or $\downarrow)$, knowing that the latter particle is located at position $\boldsymbol{r}$. While this probability is generally given by the nonlocal one-body density matrix [41], it is useful to introduce a local quantity that provides information about the short-range behavior. To this end, Becke and Edgecombe [41] introduced the local measure of fermion localization, which-in the nonrelativistic case — can be written as

$$
\mathcal{C}_{q \sigma}(\boldsymbol{r})=\left[1+\left(\frac{\tau_{q \sigma} \rho_{q \sigma}-\frac{1}{4}\left|\boldsymbol{\nabla} \rho_{q \sigma}\right|^{2}-\boldsymbol{j}_{q \sigma}^{2}}{\rho_{q \sigma} \tau_{q \sigma}^{\mathrm{TF}}}\right)^{2}\right]^{-1},
$$

where $\rho_{q \sigma}, \tau_{q \sigma}, \boldsymbol{j}_{q \sigma}$, and $\boldsymbol{\nabla} \rho_{q \sigma}$ are the particle density, kinetic energy density, current density, and density gradient, respectively. $\tau_{q \sigma}^{\mathrm{TF}}$ denotes the Thomas-Fermi kinetic energy. In this work, time reversal symmetry is conserved; hence, $\boldsymbol{j}_{q \sigma}$ vanishes.

The localization function takes generally values between 0 and 1 . A value close to 1 indicates that the probability of finding two particles (of the same type) close to each other is very low. Thus, a high value of $\mathcal{C}$ marks the spatial regions corresponding to shell separations. Since the localization function (1) is normalized to the ThomasFermi kinetic energy, $\mathcal{C}=1 / 2$ corresponds to the limit of the uniform-density Fermi gas, in which the individual orbits are spatially delocalized.

Electron localization.-For the electronic structure calculations, we used the ELF module as implemented in the relativistic ab initio quantum chemistry program DIRAC15 [52]. Hartree-Fock one-particle densities were generated in nonrelativistic, scalar-relativistic (module X2C-spinfree) [53,54], and (four-component) Dirac-Coulomb calculations in conjunction with an uncontracted relativistic quadruplezeta basis set DYALL.ACV4Z [55]. The Dirac-Fock computations include the small-component integrals as well as the two-electron Gaunt term. We utilized the finite-field method to compute the static electric dipole polarizability of $\mathrm{Og}$ (with external electric field strengths of 0.0, 0.0005, and 0.001 a.u.) at the $\operatorname{CCSD}(\mathrm{T})$ coupled-cluster level [12], which included excitations from singles, doubles, and perturbative triples. In the correlation treatment, we included 50 electrons and virtual orbitals up to 25 a.u. Here we used the molecular meanfield X2C Hamiltonian [56] with the Gaunt term included. Fock-space coupled-cluster calculations [16] were carried out to obtain the ionization potentials from the filled $7 p_{3 / 2}$ and $7 p_{1 / 2}$ shells of $\mathrm{Og}$. Note that only large-component densities are considered for the nonrelativistic and scalarrelativistic ELF, whereas in the four-component case the small-component densities are added to the large components to yield the total one-particle density. Relativistic effects make a huge imprint on many properties of Og. For instance, the electron binding energy of $\mathrm{Og}$ is predicted to rise by as much as $227 \mathrm{keV}$ by considering relativistic effects (for comparison, a similar number for $\mathrm{Pb}$ is a mere $40 \mathrm{keV}$ ).

Figures 1 and 2 show the ELFs predicted in our calculations. As seen in Fig. 1, electron localizations for $\mathrm{Xe}$ or Rn hardly change from the nonrelativistic to the fourcomponent relativistic framework. However, for Og we see significant electron delocalization with ELF values that are much smaller compared to the nonrelativistic case, making the atomic shell structure barely recognizable. The pattern of concentric rings is a fingerprint of the underlying shell structure. The sizes of rings in the ELF reflect the radii of electron orbits in different shells; hence, they roughly scale with $n^{2}$, where $n$ is the principal quantum number [41,42]. Figure 2(b) clearly shows that the delocalization is mainly due to spin-orbit coupling and not due to scalar-relativistic effects. This results in an evenly distributed ELF with values around 0.5 in the outer shells. The valence and subvalence shells of $\mathrm{Og}$ are, therefore, smeared out like in a homogenous electron gas. Rn behaves similarly to Xe, although some delocalization through relativistic effects is already apparent.

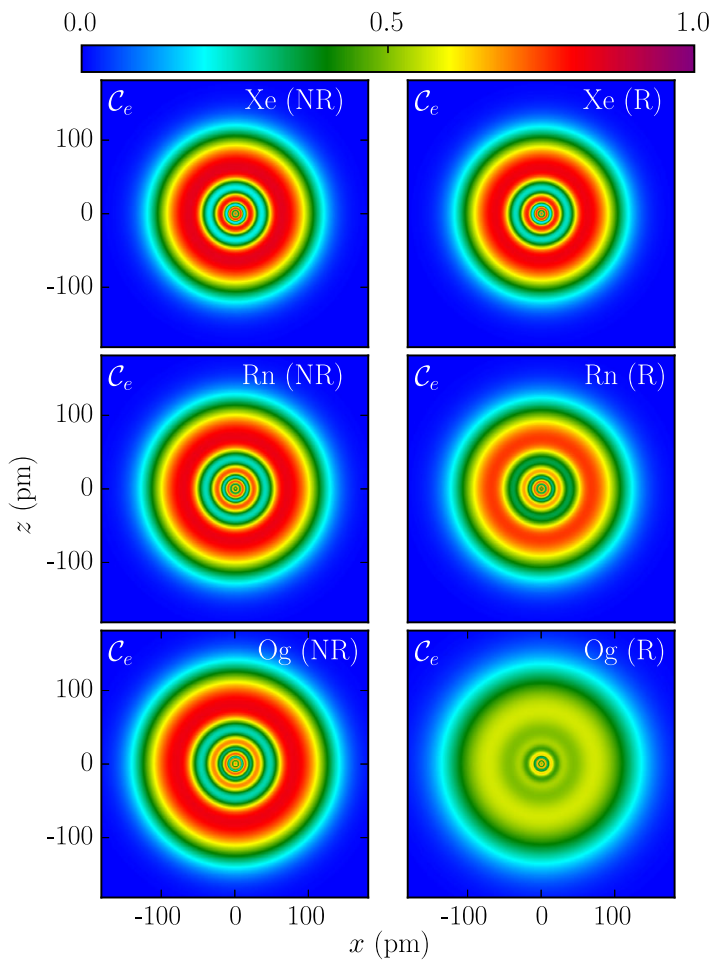

FIG. 1. ELFs from nonrelativistic (NR, left) and Dirac-HartreeFock calculations (R, right) for the heavy rare gas atoms Xe (top), Rn (middle), and Og (bottom). 


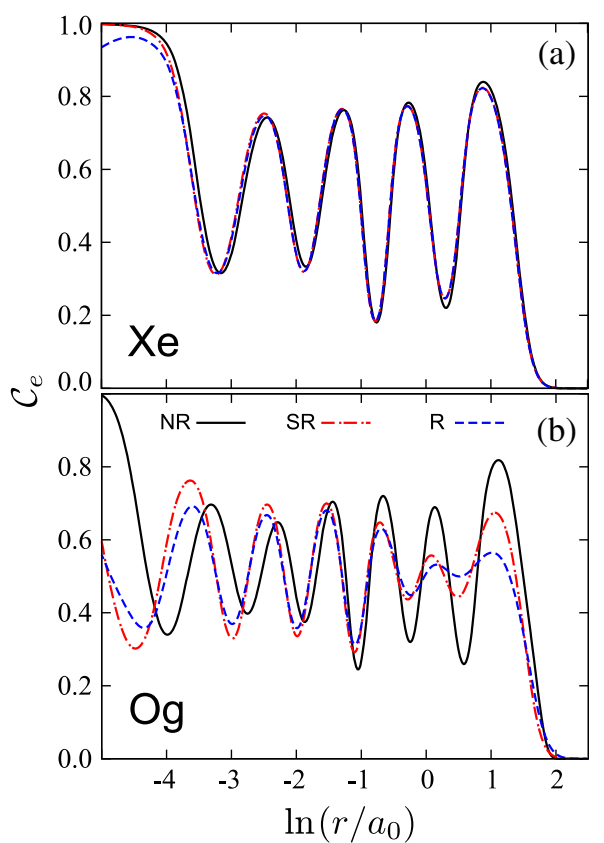

FIG. 2. ELFs for Xe (a) and Og (b) from nonrelativistic (NR), scalar-relativistic (SR), and Dirac-Hartree-Fock (R) calculations as a function of the distance from the nucleus as in Ref. [41]. The relativistic contraction of inner shells and smearing out of the shell structure in the valence and subvalence shells of Og are clearly seen.

A more detailed analysis shows that smearing out of the electron density in the valence region originates from the strong spin-orbit splitting of the $7 p$ shells; while the radii for the valence $5 p$ orbitals in Xe are very similar (2.239 and 2.141 a.u. for $5 p_{3 / 2}$ and $5 p_{1 / 2}$, respectively, as obtained with the numerical program GRASP92 [57]), the $7 p_{3 / 2}$ shell in $\mathrm{Og}$ is 0.75 a.u. further out compared to the $7 p_{1 / 2}$ shell (2.796 and 2.039 a.u., respectively). Large spin-orbit splittings are also calculated for the lower-lying $\ell>0$ (core) shells. Furthermore, the density of the single-particle (SP) states increases from Xe to Og as expected for higher principal quantum numbers; see Fig. 3. As a result of these effects, the electron density is more homogeneously distributed over the entire atomic range, i.e., less localized, resulting in ELF values oscillating around the ThomasFermi limit. Our Fock-space coupled-cluster calculation gave ionization potentials of $7 p_{3 / 2}$ and $7 p_{1 / 2}$ of 8.842 and $18.967 \mathrm{eV}$, respectively; thus, spin-orbit splitting for the valence $7 p$ orbital of $\mathrm{Og}$ is extremely large $(10.125 \mathrm{eV})$. Figure 3 illustrates this in relation to the orbital energy levels of the lighter homologues.

According to the Thomas-Fermi model, the static dipole polarizability $\alpha \propto r_{a}^{3}$, with $r_{a}^{3}$ being the atomic radius [58]. Our state-of-the-art calculations show that the electron-gaslike outer shell of $\mathrm{Og}$, resulting in $\alpha=57.98$ a.u., is much easier to polarize as compared to xenon $(\alpha=27.815$ a.u. [59]) or radon ( $\alpha=33.18$ a.u. [60]). For comparison, the nonrelativistic and scalar-relativistic values for $\mathrm{Og}$ are

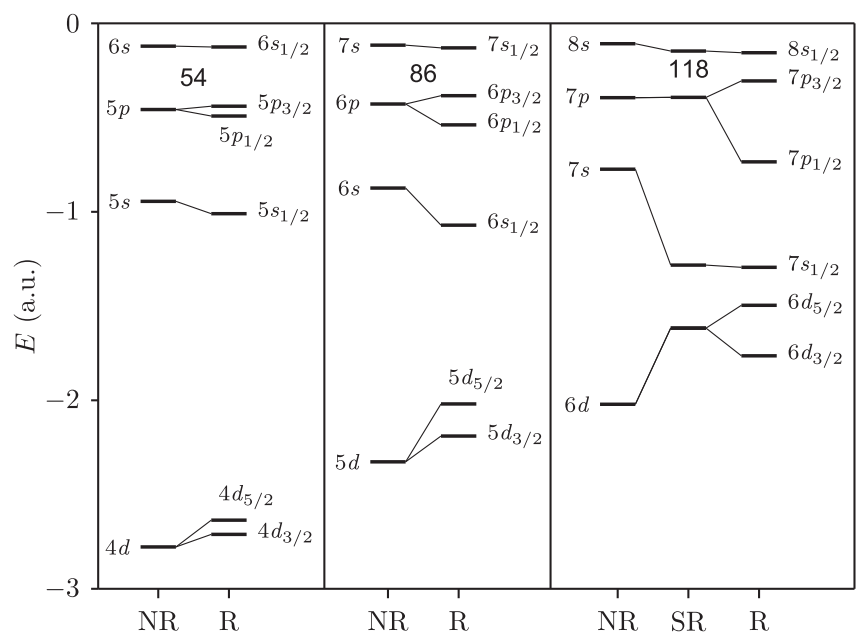

FIG. 3. Orbital energy levels of Xe (left), Rn (middle), and Og (right) for the ${ }^{1} S_{0}$ ground state as obtained from nonrelativistic (NR) and scalar-relativistic (SR) Hartree-Fock and Dirac-HartreeFock $(\mathrm{R})$ calculations. $6 s(\mathrm{Xe}), 7 s(\mathrm{Rn})$, and $8 s(\mathrm{Og})$ orbital energies are taken from the first excited ${ }^{3} P_{2}$ state.

$\alpha=45.30$ a.u. and $\alpha=43.78$ a.u., respectively. Thus, for $\mathrm{Og}$ one expects an increase in van der Waals interactions compared to the lighter rare gases and, subsequently, a significant change in the chemical and physical properties of this element; see also Refs. [10-12,15,19,61] for more discussion on this point.

Nucleon localization.-For the nuclear calculations, we employ the nuclear density functional theory (DFT) [62] with carefully optimized global Skyrme energy density functionals UNEDF1 [63] and SV-min [64]. Pairing is of minor importance in the closed-shell nuclei considered. It is treated as in Ref. [26]. Namely, we consider the densitydependent contact force at the level of the BCS theory. The pairing space is limited by a soft cutoff with the cutoff parameter chosen such that it covers about 1.6 extra oscillator shells above the Fermi energy. We use the DFT solver of Ref. [65] constrained to spherical geometry, as all nuclei considered are expected to be spherical in their ground states.

Figure 4 shows the NLFs for the doubly magic mediummass nucleus ${ }^{132} \mathrm{Sn}$ and spherical superheavy systems ${ }^{302} \mathrm{Og}$ and ${ }^{472} 164$. We consider the latter "theoretical" nucleus to further illustrate the behavior of NLFs at still larger numbers of nucleons. In contrast to the ELFs, the number of closed shells cannot be determined from the number of radial maxima. This is due to the different radial behavior of single-nucleon orbits. While the radii of electron orbits in atoms belonging to different shells are spatially well separated, the radii of nucleonic orbits scale roughly as $\sim \sqrt{2 n_{r}+\ell}$; i.e., they very gradually increase with the shell number. This results in a large spatial overlap between single-nucleon wave functions and reduced localizations as compared to the electronic case. A characteristic feature of 


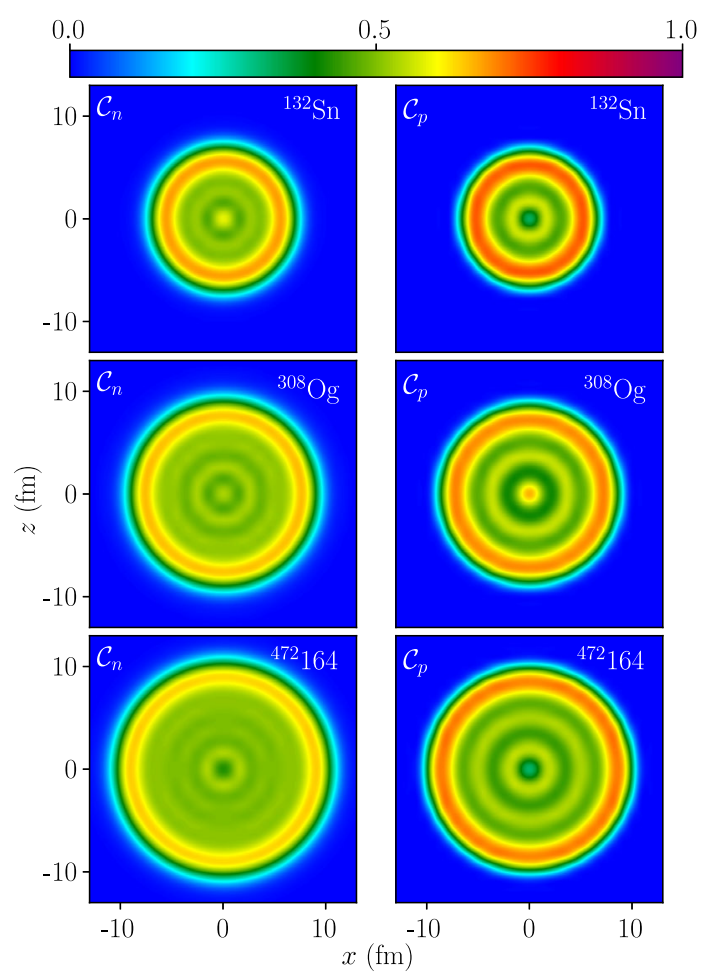

FIG. 4. NLFs of ${ }^{132} \mathrm{Sn},{ }^{302} \mathrm{Og}$, and ${ }^{472} 164$ calculated with the energy density functional UNEDF1.

NLFs is the local enhancement at the surface [49] due to the fact that few valence nucleons contribute to the total density at distances greater than the nuclear radius.

Inspecting the NLFs of protons to neutrons, one notes that the patterns of concentric rings are more distinct in the proton system, as the number of occupied proton shells is less than that for the neutrons, within the same volume (as the rms proton and neutron radii are very similar [24]). This effect becomes fairly pronounced for superheavy nuclei where the neutron excess is large. While the NLF for the medium-mass nucleus ${ }^{132} \mathrm{Sn}$ exhibits a clear shell structure with distinct oscillations around $\mathcal{C}=0.5$ [49], the maxima and minima become fainter for heavier systems. This is particularly striking for the neutrons. While the neutron $\mathrm{NLF}$ for ${ }^{302} \mathrm{Og}$ still exhibits a faint structure in the interior, the ring pattern almost vanishes for ${ }^{472} 164$. Overall, as the mass increases, the neutron localization approaches the Thomas-Fermi limit $\mathcal{C}=0.5$ in the valence region $(r>3 \mathrm{fm})$ below the surface peak. The NLF pattern seen in Fig. 4 reflects the underlying nucleonic shell structure. As discussed in, e.g., Refs. [27-32], the general pattern of SP energies undergoes significant changes in superheavy nuclei. First, the SP level density is large; in fact, it grows faster than $A^{1 / 3}$ [32]. Consequently-similar to what has been discussed earlier in the context of atomic calculations of the electron shell structure of superheavy elementssmall changes in the theoretical description can impact the shell structure substantially. Second, the shell structure of superheavy nuclei is influenced by the self-consistent interplay between the short-range attractive nuclear force and the long-range electrostatic repulsion. Thanks to the resulting Coulomb frustration, significant rearrangements of nucleonic densities, such as the appearance of central depression, are predicted [26,28,31,33-37]. The presence of central depression strongly affects high- $j$ orbits due to their large SP radii $[27,31,34,36]$.

Conclusions.-To study electronic and nucleonic shell structure in superheavy elements, we employed the local spatial measure of fermion localization. The atomic calculations were carried out for heavy rare gas atoms $\mathrm{Xe}, \mathrm{Rn}$, and the superheavy element Og recently added to the periodic table. The nuclear calculations were performed for the known doubly magic system ${ }^{132} \mathrm{Sn}$ and for superheavy nuclei ${ }^{302} \mathrm{Og}$, and ${ }^{472} 164$. This study constitutes the first application of fermion localization to superheavy atoms and nuclei.

Relativistic effects significantly impact the electronic structure of superheavy atoms. For the element Og, the electron shells with $\ell>0$ show very large spin-orbit splittings smearing out of the one-particle density, thus becoming more uniformly distributed over the entire atom approaching the electron-gas regime in the valence region. A direct consequence of this transition is its predicted large static dipole polarizability resulting in an increase in van der Waals interactions compared to the lighter rare gases and a significant change in its chemical and physical properties.

A gradual transition towards the uniform-gas regime is predicted for nucleonic localizations in superheavy nuclei. In general, neutrons are more delocalized than protons; as for the superheavy nuclei $N$ is much greater than $Z$; i.e., more neutrons are confined to the same volume than protons. While the semiclassical Thomas-Fermi limit in nuclei is strictly approached only for systems with extremely large particle numbers $A>5000$ [40,66,67], we can see that in the discussed superheavy nuclei the Fermi-gas limit of neutron NLFs is reached in the valence region $(r>3 \mathrm{fm})$ below the surface peak.

In summary, through electron and nucleon localization functions we show that $\mathrm{Og}$ is a rather unusual addition to the periodic table and to the chart of nuclides. A high density of electronic and nucleonic SP states, relativistic effects resulting in the strong spin-orbit splitting of electronic levels, and nucleonic polarization effects make the superheavy atoms, such as $\mathrm{Og}$, quantitatively different from the lighter congeners.

We acknowledge financial support by the Alexander-vonHumboldt Foundation (Bonn, Germany) and the Marsden Fund of the Royal Society of New Zealand. This work was also supported by the U.S. Department of Energy, under Awards No. DOE-DE-NA0002847 (NNSA, the Stewardship Science Academic Alliances program), No. DE-SC0013365, and No. DE-SC0008511 (Office of Science). 
[1] P. J. Karol, R. C. Barber, B. M. Sherrill, E. Vardaci, and T. Yamazaki, Pure Appl. Chem. 88, 155 (2016).

[2] Y. T. Oganessian et al., Phys. Rev. C 74, 044602 (2006).

[3] Y. Oganessian, Pure Appl. Chem. 78, 889 (2006).

[4] C. E. Düllmann, Nucl. Phys. News 27, 14 (2017).

[5] A. Türler, R. Eichler, and A. Yakushev, Nucl. Phys. A944, 640 (2015).

[6] K. S. Pitzer, J. Chem. Soc., Chem. Commun. 0, 760 b (1975).

[7] K. S. Pitzer, J. Chem. Phys. 63, 1032 (1975).

[8] P. Pyykkö, Chem. Rev. 88, 563 (1988).

[9] C. S. Nash and B. E. Bursten, Angew. Chem., Int. Ed. 38, 151 (1999).

[10] I. Goidenko, L. Labzowsky, E. Eliav, U. Kaldor, and P. Pyykkö, Phys. Rev. A 67, 020102 (2003).

[11] C. S. Nash, J. Phys. Chem. A 109, 3493 (2005).

[12] V. Pershina, A. Borschevsky, E. Eliav, and U. Kaldor, J. Chem. Phys. 129, 144106 (2008).

[13] P. Pyykkö, Phys. Chem. Chem. Phys. 13, 161 (2011).

[14] O. Kullie and T. Saue, Chem. Phys. 395, 54 (2012).

[15] A. Türler and V. Pershina, Chem. Rev. 113, 1237 (2013).

[16] E. Eliav, S. Fritzsche, and U. Kaldor, Nucl. Phys. A944, 518 (2015).

[17] P. Schwerdtfeger, L. F. Pašteka, A. Punnett, and P. O. Bowman, Nucl. Phys. A944, 551 (2015).

[18] V. Pershina, Nucl. Phys. A944, 578 (2015).

[19] A. Shee, S. Knecht, and T. Saue, Phys. Chem. Chem. Phys. 17, 10978 (2015).

[20] J. P. Desclaux, At. Data Nucl. Data Tables 12, 311 (1973).

[21] P. Indelicato, J. P. Santos, S. Boucard, and J.-P. Desclaux, Eur. Phys. J. D 45, 155 (2007).

[22] E. Eliav, U. Kaldor, Y. Ishikawa, and P. Pyykkö, Phys. Rev. Lett. 77, 5350 (1996).

[23] S. Ćwiok, P.-H. Heenen, and W. Nazarewicz, Nature (London) 433, 705 (2005).

[24] J. Erler, N. Birge, M. Kortelainen, W. Nazarewicz, E. Olsen, A. M. Perhac, and M. Stoitsov, Nature (London) 486, 509 (2012).

[25] P.-H. Heenen, J. Skalski, A. Staszczak, and D. Vretenar, Nucl. Phys. A944, 415 (2015).

[26] B. Schuetrumpf, W. Nazarewicz, and P.-G. Reinhard, Phys. Rev. C 96, 024306 (2017).

[27] S. Ćwiok, J. Dobaczewski, P.-H. Heenen, P. Magierski, and W. Nazarewicz, Nucl. Phys. A611, 211 (1996).

[28] M. Bender, K. Rutz, P.-G. Reinhard, J. A. Maruhn, and W. Greiner, Phys. Rev. C 60, 034304 (1999).

[29] M. Bender, W. Nazarewicz, and P.-G. Reinhard, Phys. Lett. B 515, 42 (2001).

[30] J. Berger, D. Hirata, and M. Girod, Acta Phys. Pol. B 34, 1909 (2003).

[31] A. V. Afanasjev and S. Frauendorf, Phys. Rev. C 71, 024308 (2005).

[32] S. E. Agbemava, A. V. Afanasjev, T. Nakatsukasa, and P. Ring, Phys. Rev. C 92, 054310 (2015).

[33] P. Möller, J. Nix, W. D. Myers, and W. J. Swiatecki, Nucl. Phys. A536, 61 (1992).

[34] J. Dechargé, J.-F. Berger, K. Dietrich, and M. S. Weiss, Phys. Lett. B 451, 275 (1999).

[35] J.-F. Berger, L. Bitaud, J. Dechargé, M. Girod, and K. Dietrich, Nucl. Phys. A685, 1 (2001).

[36] J. Dechargé, J. F. Berger, M. Girod, and K. Dietrich, Nucl. Phys. A716, 55 (2003).
[37] J. C. Pei, F. R. Xu, and P. D. Stevenson, Phys. Rev. C 71, 034302 (2005).

[38] P. Ring and P. Schuck, The Nuclear Many-Body Problem (Springer-Verlag, New York, 2000).

[39] A. Bohr and B. Mottelson, Nuclear Structure (Benjamin, New York, 1975), Vol. II.

[40] M. Brack and R. Bhaduri, Semiclassical Physics (AddisonWesley, Reading, MA, 1997).

[41] A. D. Becke and K. E. Edgecombe, J. Chem. Phys. 92, 5397 (1990).

[42] M. Kohout and A. Savin, Int. J. Quantum Chem. 60, 875 (1996).

[43] A. Savin, R. Nesper, S. Wengert, and T. F. Fässler, Angew. Chem., Int. Ed. Engl. 36, 1808 (1997).

[44] A. Scemama, P. Chaquin, and M. Caffarel, J. Chem. Phys. 121, 1725 (2004).

[45] M. Kohout, Int. J. Quantum Chem. 97, 651 (2004).

[46] T. Burnus, M. A. L. Marques, and E. K. U. Gross, Phys. Rev. A 71, 010501 (2005).

[47] J. Poater, M. Duran, M. Solà, and B. Silvi, Chem. Rev. 105, 3911 (2005).

[48] P.-G. Reinhard, J.A. Maruhn, A. S. Umar, and V.E. Oberacker, Phys. Rev. C 83, 034312 (2011).

[49] C. L. Zhang, B. Schuetrumpf, and W. Nazarewicz, Phys. Rev. C 94, 064323 (2016).

[50] B. Schuetrumpf and W. Nazarewicz (to be published).

[51] B. Schuetrumpf, C. Zhang, and W. Nazarewicz, in Nuclear Particle Correlations and Cluster Physics (World Scientific, Singapore, 2017), Chap. 5, p. 135.

[52] DIRAC, a relativistic ab initio electronic structure program, Release DIRAC15 (2015), written by R. Bast et al. (see http://www.diracprogram.org).

[53] K. G. Dyall, J. Chem. Phys. 100, 2118 (1994).

[54] M. Iliaš and T. Saue, J. Chem. Phys. 126, 064102 (2007).

[55] K. G. Dyall, Theor. Chem. Acc. 115, 441 (2006).

[56] J. Sikkema, L. Visscher, T. Saue, and M. Iliaš, J. Chem. Phys. 131, 124116 (2009).

[57] K. G. Dyall, I. P. Grant, F. A. Parpia, and E. P. Plummer, Comput. Phys. Commun. 55, 425 (1989).

[58] V. P. Shevelko and A. V. Vinogradov, Phys. Scr. 19, 275 (1979).

[59] U. Hohm and K. Kerl, Mol. Phys. 69, 803 (1990).

[60] T. Nakajima and K. Hirao, Chem. Lett. 30, 766 (2001).

[61] T. Hangele and M. Dolg, J. Chem. Phys. 138, 044104 (2013).

[62] M. Bender, P.-H. Heenen, and P.-G. Reinhard, Rev. Mod. Phys. 75, 121 (2003).

[63] M. Kortelainen, J. McDonnell, W. Nazarewicz, P.-G. Reinhard, J. Sarich, N. Schunck, M. V. Stoitsov, and S. M. Wild, Phys. Rev. C 85, 024304 (2012).

[64] P. Klüpfel, P. G. Reinhard, T. J. Bürvenich, and J. A. Maruhn, Phys. Rev. C 79, 034310 (2009).

[65] P.-G. Reinhard, in Computational Nuclear Physics I-Nuclear Structure, edited by K. Langanke, S. Koonin, and J. Maruhn (Springer, Berlin, 1991), p. 28.

[66] O. Bohigas, X. Campi, H. Krivine, and J. Treiner, Phys. Lett. B 64, 381 (1976).

[67] P.-G. Reinhard, M. Bender, W. Nazarewicz, and T. Vertse, Phys. Rev. C 73, 014309 (2006).

Correction: The previously published Figure 2 contained an error in the axis label and has been replaced. 